\title{
Indirect versus direct detection methods of Trichinella spp. infection in wild boar (Sus scrofa)
}

\author{
Maria Angeles Gómez-Morales ${ }^{1 *}$, Alessandra Ludovisi ${ }^{1}$, Marco Amati ${ }^{1}$, Ennio Bandino², Gioia Capelli ${ }^{3}$, \\ Franco Corrias ${ }^{4}$, Luca Gelmini ${ }^{5}$, Alberigo Nardi ${ }^{6}$, Cristina Sacchi ${ }^{7}$, Simona Cherchi ${ }^{1}$, Marco Lalle ${ }^{1}$ \\ and Edoardo Pozio ${ }^{1}$
}

\begin{abstract}
Background: Trichinella spp. infections in wild boar (Sus scrofa), one of the main sources of human trichinellosis, continue to represent a public health problem. The detection of Trichinella spp. larvae in muscles of wild boar by digestion can prevent the occurrence of clinical trichinellosis in humans. However, the analytical sensitivity of digestion in the detection process is dependent on the quantity of tested muscle. Consequently, large quantities of muscle have to be digested to warrant surveillance programs, or more sensitive tests need to be employed. The use of indirect detection methods, such as the ELISA to detect Trichinella spp. infections in wild boar has limitations due to its low specificity. The aim of the study was to implement serological detection of anti-Trichinella spp. antibodies in meat juices from hunted wild boar for the surveillance of Trichinella spp. infections.
\end{abstract}

Methods: Two tests were used, ELISA for the initial screening test, and a specific and sensitive Western blot (Wb) as a confirmatory test. The circulation of anti-Trichinella lgG was determined in hunted wild boar muscle juice samples in 9 provinces of 5 Italian regions.

Results: From 1,462 muscle fluid samples, 315 (21.5\%, 95\% C.I. 19.51-23.73) were tested positive by ELISA. The 315 ELISA-positive muscle fluid samples were further tested by Wb and 32 (10.1\%, 95\% C.I. 7.29-13.99) of these were positive with a final seroprevalence of 2.2\% (95\% C.I 1.55-3.07; 32/1,462). Trichinella britovi larvae were detected by artificial digestion in muscle tissues of one $(0.07 \%, 95 \%$ C.I. $0.01-0.39)$ out of the 1,462 hunted wild boars. No Trichinella spp. larvae were detected in Wb-negative wild boar. From 2006 to 2012, a prevalence of $0.017 \%$ was detected by muscle digestion in wild boar hunted in the whole Italian territory.

Conclusions: The combined use of both serological methods had a sensitivity 31.4 times higher than that of the digestion $(32 / 1,462$ versus 1/1,462), suggesting their potential use for the surveillance of the Trichinella spp. infection in wild boar populations.

Keywords: Trichinella spp, ELISA, Western blot, Wild boar, Meat juice, Chemiluminescence, Prevalence, Surveillance, Italy

\section{Background}

Wild carnivore and omnivore (mainly swine) animals are the main reservoir of nematodes of the genus Trichinella, aetiological agents of trichinellosis, a serious and sometimes fatal zoonotic disease [1]. These parasites circulate in all continents with the exception of the Antarctica. In the last 70 years, there has been increasing evidence that

\footnotetext{
* Correspondence: mariaangeles.gomezmorales@iss.it

${ }^{1}$ Department of Infectious, parasitic and immunomediated Diseases, Istituto

Superiore di Sanità, Viale Regina Elena 299, 00161 Rome, Italy

Full list of author information is available at the end of the article
}

the biomass of nematodes of the genus Trichinella is greater in wild animals than in domestic animals [2].

Since wild boar (Sus scrofa) is frequently consumed by humans, the presence of Trichinella spp. in this animal represents a threat for human health [3]. Consequently in the European Union, both bred and hunted wild boar for the market is systematically sampled in slaughterhouses or game-handling establishments to detect Trichinella spp. larvae by muscle digestion [4]. However, wild boar for private consumption is exempt from the official controls in some member countries, with the 
result that these animals bypass veterinary inspection. Consequently, Trichinella spp. surveillance programs should be implemented for wild boar in terms of food safety and public awareness increased by informing about the possible risk of acquiring trichinellosis.

For Italian wildlife, the most prevalent species is Trichinella britovi, which infect carnivore mammals such as red fox, wolf, and mustelids [5-7]. This parasite species has also been detected in wild boar despite its low prevalence $(0.006 \%-0.017 \%)$ [6,8-14].

Serological methods are suitable for surveillance and epidemiological investigations in swine populations [15] and are considered as an appropriate tool for monitoring programs once they are validated by an independent body [4]. ELISA can detect Trichinella-specific antibodies in serum and meat juice samples [16], showing a high sensitivity, but may also result in a low specificity due to falsepositive reactions especially among swine, which are not reared under controlled conditions $[15,17,18]$.

Therefore, confirmatory testing is required to substantiate the ELISA results [19]. In this regard, several Trichinella spiralis proteins recognized by pig sera have been identified by western blotting (Wb) using excretory/secretory antigens (ESA) or a crude worm extract [18-23]. Recently, a distinctive triple-band Wb pattern of Trichinella spp. infection has been defined for pigs. However, the visual interpretation of the band pattern is not exempt of problems [18].

The aim of the study was to implement the serological detection of anti-Trichinella spp. antibodies in muscle juices from hunted wild boar for the surveillance of Trichinella spp. infections. Two tests were used, ELISA for an initial screening test and a specific $\mathrm{Wb}$ as confirmatory test. To increase the accuracy of the $\mathrm{Wb}$, a high sensitive revelation system (chemiluminescence) and image software analysis were used; the serological results were then compared with those from the muscle tissue digestion.

\section{Methods}

\section{Sample collection}

During the October 2007 - January 2008 hunting season, diaphragm muscles were collected from wild boar hunted in 9 provinces of 5 regions of northern and central Italy and from Sardinia, selected on the basis of information relating to the presence/absence of Trichinella spp. in wildlife [7]: 1) the Alpine area of the Lombardy region (northern Italy) where $T$. britovi has been frequently detected; 2) the Euganean Hills, a group of isolated hills (Veneto region, north-eastern Italy), where Trichinella spp. have never been documented, as well as in the whole of the Veneto region after 2005; 3) the Apennine area of the Emilia-Romagna region (northern Italy), where both $T$. britovi and T. pseudospiralis have been documented; 4) the Apennine area, province of Florence (Tuscany region, central Italy) where Trichinella spp. have never been documented, unlike its bordering provinces where $T$. britovi has been documented; 5) the Grosseto province (Tuscany region), where T. pseudospiralis has been recently documented in a wild boar; and 6) the mountain area of the Nuoro province in Sardinia where $T$. britovi has been documented among freeranging pigs since 2005 to the present [24] (Figure 1).

Diaphragm muscles (50-100 g) were collected from 1,462 wild boars immediately after a hunt, preserved in plastic bags, refrigerated in an ice box during transportation from the field to the laboratory, then again refrigerated at $4^{\circ} \mathrm{C}$ until digestion, which was performed within 24-48 h after the hunt. Before digestion, muscle was cut into small $5 \mathrm{~g}$ pieces and was digested according to the Commission Regulation 2075/2005 [4]. The recovered larvae were counted under a stereomicroscope and the number of larvae per gram (LPG) was determined. The remaining muscle was frozen at $-20^{\circ} \mathrm{C}$. The frozen muscle was shipped on dry ice from the peripheral laboratories to the Istituto Superiore di Sanità (Rome, Italy). On arrival, the frozen muscle was stored at $-20^{\circ} \mathrm{C}$ until processing. The day before running the serological test, muscle samples were thawed at room temperature and the muscle fluids were collected from the plastic bags and aliquoted at $0.3 \mathrm{ml}$ per vial.

Muscle samples were also collected from 302 pigs from herds kept under controlled management conditions (negative controls). For each animal, $10 \mathrm{~g}$ of diaphragm muscle was digested according to a published protocol, as previously mentioned [4]. Muscle fluids from 4 experimentally infected (20,000 larvae) pigs were used as positive controls.

\section{Trichinella sp. larva identification}

After digestion, Trichinella spp. larvae were washed in a phosphate buffer saline solution, counted in triplicate, and stored in $90 \%$ ethyl alcohol for their molecular identification. Single Trichinella spp. larvae were identified at the species level by a multiplex PCR analysis, performed according to a validated protocol at the European Union Reference Laboratory for Parasites (Rome, Italy) [25].

\section{ELISA}

The 1/10 diluted muscle fluids from wild boar and control pigs were first tested for the presence of anti-Trichinella IgG by ELISA using excretory/secretory antigens (ESA). An in-house ELISA was used in accordance with a previously published validated protocol $[17,18]$. Since raw optical density (OD) values are absolute measurements that are influenced by ambient temperature, test parameters, and photometric instruments, the results were expressed as a function of the reactivity of the 


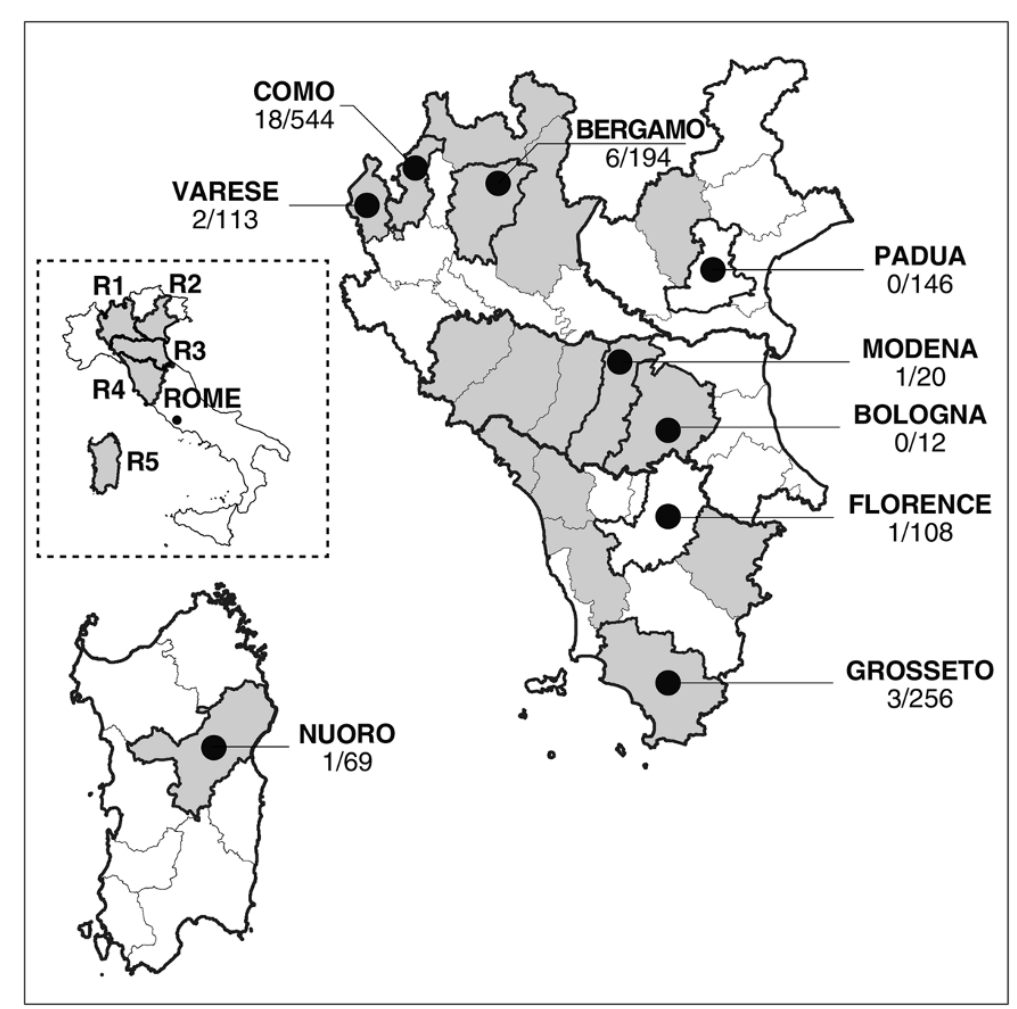

Figure 1 Western blot positive/tested wild boar (Sus scrofa) by Italian provinces. Black dots, Italian provinces. R1, Lombardy region; R2, Veneto region; R3, Emilia Romagna region; R4, Tuscany region; and R5, Sardinia region. Grey area, indicates the provinces where Trichinella spp. have been previously documented in wildlife; white area, the provinces where Trichinella spp. have never been documented in wildlife and/or domestic animals in the last 50 years.

positive control muscle fluid sample with the highest value out of the 4 included in each run of the assay. This control must yield a result that is in the linear range of the measurement [26]. The mean OD values of the control muscle fluids, as well as the mean OD values of the duplicate test muscle fluids, were then calculated, and for each muscle fluid an ELISA index $\left(\mathrm{I}_{\mathrm{E}}\right)$ expressed as percentage of positivity was calculated according to the following equation:

$$
I_{E}=\frac{\text { mean OD value of duplicate sample-OD blank }}{\text { mean OD value of the highest positive control-OD blank }} \times 100
$$

The cut-off value for OD, calculated as the mean $( \pm 3$ SD) of the OD values of the 302 muscle fluids from Trichinella-negative pigs was $24.8 \%$.

\section{Western blot}

All ELISA-positive muscle fluids, were then tested by $\mathrm{Wb}$ according to a previously published validated protocol [18], but using a high sensitive revelation system based on chemiluminescence, as the level of antibodies in muscle fluids is lower than that in sera [16]. Furthermore, to assess the quality of the electrophoretic transfer of gels, a pre-stained standard of molecular weight was used (Precision Plus Protein ${ }^{\mathrm{Tm}}$ WesternC ${ }^{\mathrm{Tm}}$ Standars, BioRad, Hercules, CA, USA) in each run. An experiment was considered to be valid when all of the pre-stained protein standards $(250,150,100,75,50,37,25$ and 20 $\mathrm{kD})$ were separated and transferred onto the nitrocellulose membrane, and the relative mobility of each standard was within the standard range previously established by 3 independent experiments. The nitrocellulose filters were blocked with $5 \%$ skimmed milk in $1 \mathrm{X}$ Tris-Borate saline-Tween (TBST, $50 \mathrm{mM}$ Tris $\mathrm{pH}$ 8.0, $150 \mathrm{~m} \mathrm{NaCL}$, $1 \%$ Tween 20 ) at $4^{\circ} \mathrm{C}$ overnight and washed 3 times with $1 \mathrm{X}$ TBST. Each nitrocellulose filter was cut into strips, each of which was then incubated with $1 / 10$ muscle fluid with $3 \%(\mathrm{w} / \mathrm{v})$ skimmed milk (Sigma-Aldrich, Saint Louis, MO, USA) in $1 \mathrm{X}$ TBST at RT for $1 \mathrm{~h}$. After washing 3 times with $1 \mathrm{X}$ TBST, the pre-stained protein standard strip was incubated with Precision Protein ${ }^{\text {ma }}$ Strep Tactin-HRP conjugated at 1:10,000 dilution, for $1 \mathrm{~h}$. The other strips (previously incubated with the muscle fluid) were incubated with a 1:5,000 dilution of goat anti-swine IgG conjugated with horseradish peroxidase (Bio-Rad, Hercules, CA, USA) for $1 \mathrm{~h}$. To reveal proteins with high efficiency, the LiteAblot ${ }^{\circledR}$ Plus chemiluminescence system (Euroclone, Pero, Milan, Italy) was 
added to the strips for $5 \mathrm{~min}$. The proteins were then visualized on a ChemiDoc ${ }^{\mathrm{TM}}$ XRS System (Bio-Rad) and images were analyzed using the Image $\mathrm{Lab}^{\mathrm{Tm}}$ software version 4.0 (Bio-Rad). The experiment was considered valid when the relative mobility (Rf) of the proteins which define the pattern of Trichinella spp. infections [18], were within the range previously established by 3 independent experiments for each positive control (first band from 0.410 to $0.524 \mathrm{~mm}$; second band from 0.365 to $0.499 \mathrm{~mm}$; and third band from 0.319 to $0.428 \mathrm{~mm}$ ). The positivity/negativity of each wild boar muscle fluid was then determined by comparing the Rf value of each sample with the positive control on the same blot, and the corresponding MW calculated by the Image Lab $^{\text {тM }}$ software version 4.0 (Bio-Rad).

\section{Statistical analysis}

Two-tailed Student's t-tests were performed using GraphPad Prism (Version 3, Graph-Pad Software, San Diego, CA, USA) to compare the test performance. The differences of prevalence obtained by serology (Wb) and digestion method were tested using the chi-square test.

\section{Ethical statement}

The wild boar meat sampling was performed according to the Commission Regulation 2075/2005 [4].

\section{Results}

\section{Artificial digestion}

Trichinella spp. larvae were detected in one $(0.07 \%, 95 \%$ C.I. 0.01-0.39) out of the 1,462 muscle samples from hunted wild boar. The Trichinella sp. positive wild boar with a larval burden of 0.15 LPG was hunted in the Varese province (Figure 1). No Trichinella sp. larva was detected in the 302 pig muscle samples from herds kept under controlled management conditions. The Trichinella sp. larvae isolated from the wild boar were identified as T. britovi (data not shown).

\section{ELISA}

As shown in Table 1 and Figure 2, out of the 1,462 muscle fluids from wild boar, 315 (21.5\%, 95\% C.I. 19.51-23.73) tested positive by ELISA $\left(173,54.9 \%\right.$, with an $\mathrm{I}_{\mathrm{E}}$ from 24.8 to $34.8 ; 84,26.8 \%$, with an $\mathrm{I}_{\mathrm{E}}$ from 34.8 to $44.8 ; 40,12.7 \%$, with an $\mathrm{I}_{\mathrm{E}}$ from 44.8 to 54.8 ; and $18,5.7 \%$, with an $\mathrm{I}_{\mathrm{E}}>$ 54.8). The wild boar which tested positive for T. britovi larvae showed an $\mathrm{I}_{\mathrm{E}}$ of 34.0. The prevalence of ELISA-positive muscle fluids ranged from $7.0 \%$ to $33.5 \%$ with respect to the province of origin (Table 1). None of the muscle fluids from the 302 control pigs tested positive by ELISA.

\section{Western blot}

The 315 ELISA-positive muscle fluids were further tested by $\mathrm{Wb}$ and 32 (10.1\%, 95\% C.I. 7.29-13.99) including the wild boar which had been tested positive by

Table 1 Prevalence of anti-Trichinella IgG detected in muscle fluids of wild boar hunted in Italy

\begin{tabular}{|c|c|c|c|c|}
\hline $\begin{array}{l}\text { Region of } \\
\text { origin province }\end{array}$ & $\begin{array}{l}\text { ELISA positive/tested } \\
\text { wild boar }(\%)\end{array}$ & $\begin{array}{l}\text { Western blot positive/ELISA } \\
\text { positive wild boar (\%) }\end{array}$ & $\begin{array}{l}\text { Western blot positive/ } \\
\text { tested wild boar (\%) }\end{array}$ & $\begin{array}{l}\text { Previous reports of } \\
\text { Trichinella spp. in wildlife }\end{array}$ \\
\hline \multicolumn{5}{|l|}{ Lombardy } \\
\hline Bergamo & 65/194 (33.5) & $6 / 65(9.2)$ & 6/194 (3.1) & Yes \\
\hline Como & $159 / 544(29.2)$ & 18/159 (11.3) & 18/544 (3.3) & Yes \\
\hline Varese & $31^{\mathrm{a} / 113}(27.4)$ & $2^{a} / 31(12.5)$ & $2^{a} / 113(1.8)$ & Yes \\
\hline \multicolumn{5}{|l|}{ Veneto } \\
\hline Padua & $21 / 146(14.4)$ & $0.0 / 21$ & $0.0 / 146$ & $\mathrm{No}^{b}$ \\
\hline \multicolumn{5}{|l|}{ Emilia Romagna } \\
\hline Bologna & $1 / 12(8.3)$ & $0.0 / 1$ & $0.0 / 12$ & Yes \\
\hline Modena & 4/20 (20.0) & $1 / 4$ & $1 / 20(5.0)$ & Yes \\
\hline \multicolumn{5}{|l|}{ Tuscany } \\
\hline Florence & 12/108 (11.1) & $1 / 12(8.3)$ & 1/108 (0.9) & $\mathrm{No}^{\mathrm{C}}$ \\
\hline Grosseto & $18 / 256(7.0)$ & $3 / 18$ (16.7) & $3 / 256(1.2)$ & Yes \\
\hline \multicolumn{5}{|l|}{ Sardinia } \\
\hline Nuoro & $5 / 69(7.2)$ & $1 / 5$ & $1 / 69(1.4)$ & $\mathrm{No}^{\mathrm{d}}$ \\
\hline Total & $315^{a} / 1,462(21.5)$ & $32^{a} / 315(10.1)$ & $32^{a} / 1,462(2.2)$ & \\
\hline
\end{tabular}

${ }^{a}$ Trichinella britovi larvae detected in muscle tissues of a single animal.

${ }^{b}$ none reported in the Padua province and Veneto region since 2005 (see Figure 3).

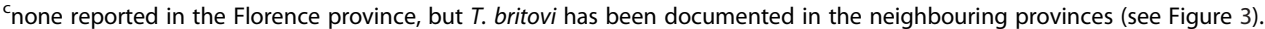

${ }^{d}$ none reported in wild animals before 2011, but $T$. britovi has been documented in free-ranging pigs from 2005 to the present. 


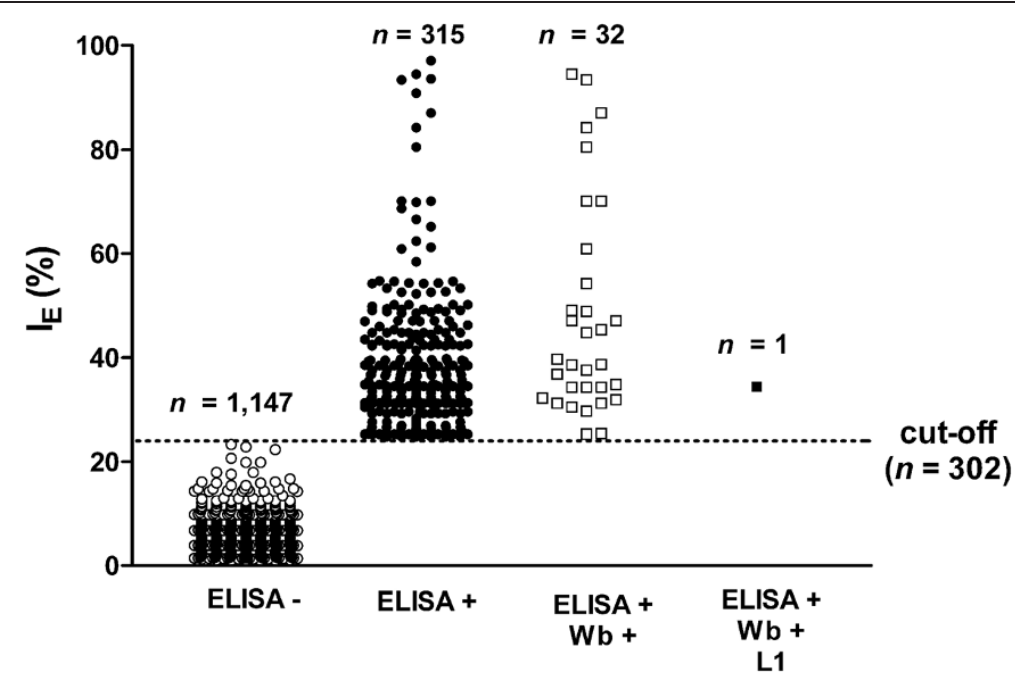

Figure 2 Scatter plot of the ELISA index ( $\left.\mathrm{I}_{\mathrm{E}}\right)$ for anti-Trichinella spp. IgG in wild boar. ELISA-, muscle fluids resulted negative by ELISA; ELISA+, muscle fluids resulted positive by ELISA; Wb+, muscle fluids resulted positive by ELISA and Western blot; L1, wild boar tested positive for Trichinella britovi larvae by artificial digestion.

digestion, showed the 3 band pattern which is considered to be diagnostic for the presence of anti-Trichinella IgG (Table 1; Figures 2 and 3). No positive muscle fluid was detected by $\mathrm{Wb}$ from wild boar hunted in the Euganean area. In the Alpine areas of the Lombardy region, a prevalence from $1.8 \%$ to $3.3 \%$ of anti-Trichinella IgG was detected by $\mathrm{Wb}$ in the wild boar population according to the province of origin (Figure 1). A prevalence of $5.0 \%$ was detected by $\mathrm{Wb}$ in wild boar hunted in the Apennine area of the Emilia Romagna region (Figure 1). A prevalence of $0.9 \%$ and of $1.2 \%$ was detected by $\mathrm{Wb}$ in wild boar hunted in the Florence and Grosseto provinces, respectively (Figure 1). The muscle fluid of 1 (1.4\%) out of 69 wild boars from Sardinia was positive for anti-Trichinella IgG by Wb (Figure 1).

\section{Discussion}

The $\mathrm{Wb}$ results show a total prevalence of anti-Trichinella IgG in the tested wild boar populations of Italy of $2.2 \%$ (95\% C.I. $1.55-3.07 ; 32 / 1,462$ ), which is 31.4 times higher than the prevalence detected by digestion of muscle tissues $(0.07 \% ; P<0.001)$. The serological prevalence varies according to the hunting areas and is consistent with the information on the circulation of these zoonotic parasites in wildlife (Figure 1) [5-7].

The detection of only a single positive wild boar $(0.07 \%)$ by the digestion test cannot be attributed to poor test performance, since all the laboratories performing the test are accredited according to ISO 17025. Additionally, a large quantity of preferential muscles from each animal was tested in two different laboratories, and a worm burden of 0.15 LPG was detected suggesting a good sensitivity.
The different prevalence obtained by digestion and serology $(0.07 \%$ versus $2.2 \%, P<0.001)$ can be explained by the analytical sensitivity of the artificial digestion test which is of 1 LPG [26-28], whereas, the analytical sensitivity of the ELISA has been reported to be 0.01 LPG $[26,27]$. No data exist on the analytical sensitivity of $\mathrm{Wb}$, namely the minimum detectable antibody level by $\mathrm{Wb}$ and the number of LPG in preferential muscles. However, the study results show that anti-Trichinella IgG can be detected by $\mathrm{Wb}$ in the muscle juice of a wild boar with 0.15 LPG suggesting a good sensitivity for this test when chemiluminescence was used. Moreover, visual observation does not permit the $\mathrm{Wb}$ band pattern to be objectively evaluated. To achieve a high accuracy in the interpretation of the $\mathrm{Wb}$ pattern, it is necessary to carefully measure the relative migration distances of the proteins to infer the molecular weight. Consequently, the interpretation of the $\mathrm{Wb}$ pattern is time consuming and needs experienced personnel. These problems can be overcome by the use in reference laboratories of an image analyzing software, which could permit the standardization of the procedure and also an accurate interpretation of the results. The serological specificity has been increased by coupling the ELISA with the $\mathrm{Wb}$, and the $\mathrm{Wb}$ sensitivity has been increased by using a high sensitive revelation system based on chemiluminescence (Figure 3).

The discordance between the prevalence obtained by serology and digestion (2.2\% versus $0.07 \%$ ) could be related also to biological and immunological reasons. Experimental infections show a low infection burden and a short survival time of $T$. britovi larvae in swine muscles. At two months post infection the number of $T$. britovi larvae in swine muscles ranges from 1.6 to 280 times 

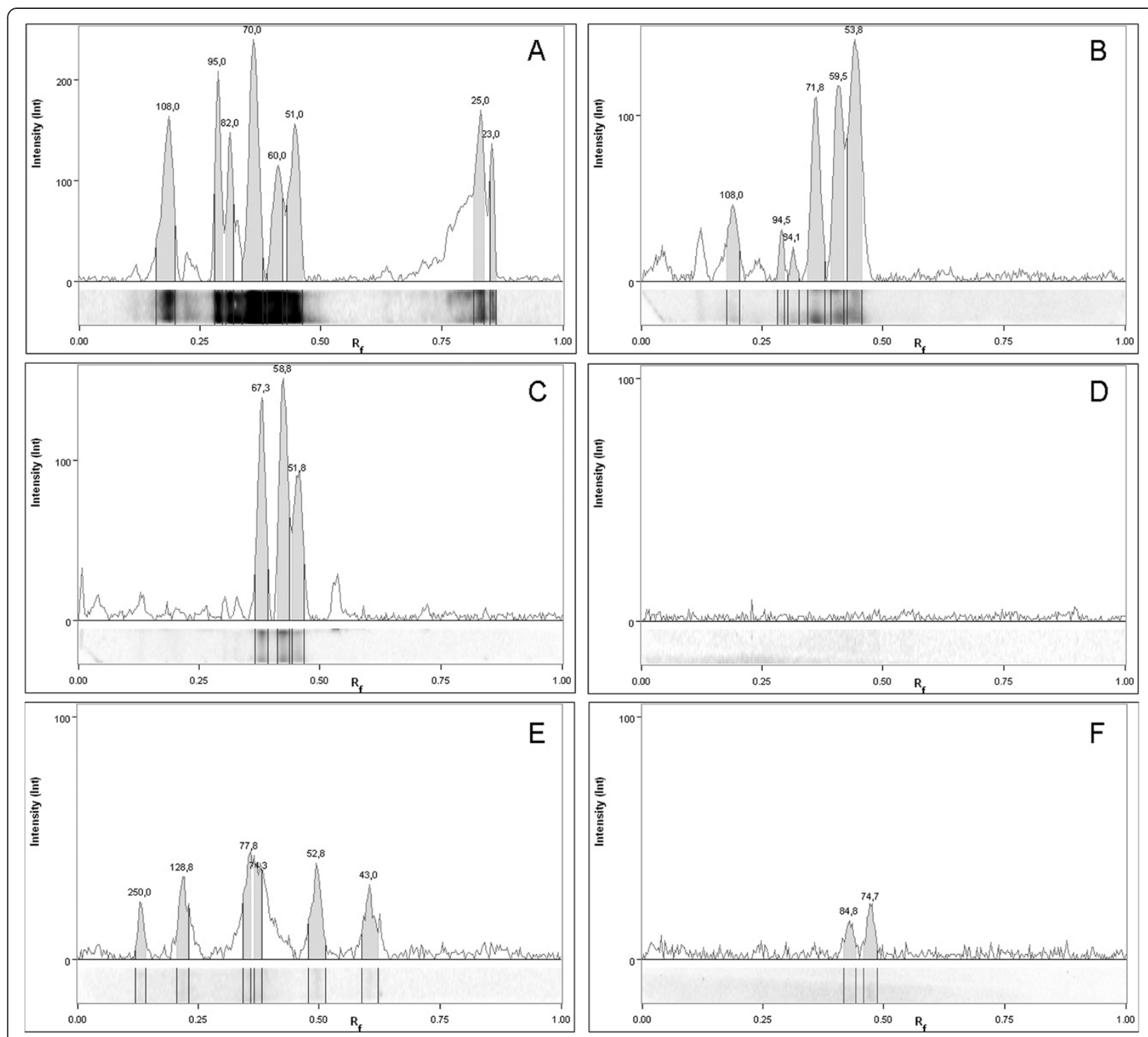

Figure 3 Trichinella spiralis excretory/secretory antigens recognized by Western blot in muscle fluids from wild boar. Signal intensities and relative migration values (Rf) of the proteins recognized by: a positive control muscle fluid from an experimentally infected pig, panel $\mathbf{A}$; the muscle fluid from the wild boar with 0.15 LPG in the diaphragm, panel B; a low positive muscle fluid from a wild boar, panel $\mathbf{C}$; a muscle fluid from an uninfected control pig, panel $\mathbf{D}$; and the false positive muscle fluids from two ELISA-positive wild boars, panels $\mathbf{E}$ and $\mathbf{F}$. Numbers above each peak refer to the software deduced molecular weights of the bands.

lower than that of T. spiralis [16]. Furthermore, reinfections cannot be excluded. A primary infection with T. spiralis in pigs induces nearly absolute resistance to the muscle establishment of larvae from new infectious worms, and the fecundity of female worms recovered from immune pigs was reduced by $75 \%$ in comparison to controls [29]. Despite the low infectivity of T. nativa for swine, primary inoculation with this species resulted in high levels of immunity against challenge infection with $T$. spiralis; this immunity was expressed in accelerated expulsion of challenge adults from the intestine and reduced numbers of muscle larvae [30].

The study results show that anti-Trichinella IgG can be easily detected in the muscle juice. Furthermore in wild animals, it is easier to collect muscle juices of good quality than to collect serum samples of good quality, namely not haemolysed and without bacteria. Once this indirect detection system (ELISA $+\mathrm{Wb}$ ) is validated in other areas with different degrees of prevalence, it could be included as a tool for the surveillance of Trichinella spp. infection in wild 
boar and in domestic pig populations at risk for these zoonotic parasites, namely backyard and free-ranging pigs [31].

Recently, a Trichinella seroprevalence of 3.5\% (95\% C.I. 0.0-8.0) was detected in sera from wild boar hunted in the Australian mainland, by an ELISA coupled with a Wb in a similar way to that used in the present study. The seroprevalence was significantly higher $(\mathrm{P}<0.05)$ than that of the artificial digestion $(0.0 \%, 95 \%$ C.I. $0.0-1.1)$ [32]. In a similar way to the results of the present study, Trichinella papuae larvae were detected in a muscle sample from only a single wild boar hunted in the Gabba Island in the Torres Strait not far from the Australia mainland. Here only serologically positive animals have been detected.

In another survey carried out on wild boar of southcentral Spain [33], a different prevalence was observed between serology and digestion, than in the present study: i) $\mathrm{Wb}$ has been performed on only a percentage of ELISA-positive sera; ii) no chemiluminescence has been used to increase the $\mathrm{Wb}$ sensitivity; and iii) both $T$. britovi and T. spiralis is known to circulate in the investigated region, the latter being more infectious for swine. In North Carolina, a serological prevalence of $13.3 \%$ was detected in feral pigs by a commercial ELISA kit, but ELISA-positive samples were not confirmed by $\mathrm{Wb}$ and no information is available on Trichinella spp. larvae in muscles [34].

Trichinella britovi was the only species detected in Italian wildlife up to few years ago when T. pseudospiralis began to be documented in northern and central Italy $[7,35]$. The prevalence of Trichinella spp. infections in the wild boar populations of Italy has been always extremely low. From 1985 to 1999, T. britovi was detected in $9(0.002 \%)$ out of 370,000 hunted wild boars [6]. In the last 13 years, there has been an increase of the wild boar population in Italy and, in parallel, an increase of the number of hunted wild boar and an increase of wild boar tested for Trichinella spp. per year according to the Commission Regulation 2075/2005 [4]. From 2007 to 2012 , a total prevalence of $0.017 \%$ (95\% CI 0.0001 0.0002; 47 positive/268,200 tested), was detected [8-14], which is significantly higher $(P<0.001)$ than the prevalence detected in the previous period, despite the great differences among the Italian regions (data not shown).

\section{Conclusions}

The highly specific and sensitive indirect detection system $($ ELISA $+\mathrm{Wb})$ used in the present work, represents a very useful tool to increase the efficacy of surveillance programs. In fact, this detection system may allow the identification of the areas where Trichinella spp. are already circulating but where no larva have yet been detected by direct methods.

\section{Competing interests}

The authors declare that they have no competing interests.

\section{Authors' contributions}

All the authors have contributed significantly to this study. MAGM and EP designed the study, performed the data analysis and interpretation, and prepared the manuscript. AL, MA, SC and ML performed laboratory tests and processed the data. EN, GC, FC, LG, AN and CS organized the sample collection on the field and the muscle digestion in the peripheral laboratories. All authors read and approved the final manuscript.

\section{Acknowledgments}

We are grateful to Marilena Interisano for the molecular identification of larvae. This work has been supported by the European Commission (contract SANCO/2006/FOODSAFETY/032).

\section{Author details}

${ }^{1}$ Department of Infectious, parasitic and immunomediated Diseases, Istituto Superiore di Sanità, Viale Regina Elena 299, 00161 Rome, Italy. ${ }^{2}$ Istituto Zooprofilattico della Sardegna, Nuoro section, Nuoro, Italy. ${ }^{3}$ Istituto Zooprofilattico Sperimentale delle Venezie, 35020 Legnaro, Italy. ${ }^{4}$ Istituto Zooprofilattico del Lazio e della Toscana, Florence section, Florence, Italy. ${ }^{5}$ Istituto Zooprofilattico della Lombardia e dell'Emilia Romagna, Modena section, Modena, Italy. ${ }^{6}$ Istituto Zooprofilattico del Lazio e della Toscana, Grosseto section, Grosseto, Italy. ${ }^{7}$ Istituto Zooprofilattico della Lombardia e dell'Emilia Romagna, Binago section, Varese, Italy.

Received: 10 February 2014 Accepted: 2 April 2014

Published: 7 April 2014

\section{References}

1. Gottstein B, Pozio E, Nöckler K: Epidemiology, diagnosis, treatment, and control of trichinellosis. Clin Microbiol Rev 2009, 22:127-145.

2. Pozio E, Zarlenga DS: New pieces of the Trichinella puzzle. Int J Parasitol 2013, 43:983-997.

3. Murrell KD, Pozio E: Worldwide occurrence and impact of human trichinellosis, 1986-2009. Emerg Infect Dis 2011, 17:2194-2202.

4. Community E: Regulation (EC) No 2075/2005 of the European Parliament and of the Council of 5 December 2005 laying down specific rules on official controls for Trichinella in meat. Off J EC L 2005, 338:60-82.

5. Pozio E: Trichinellosis in the European Union: epidemiology, ecology and economic impact. Parasitol Today 1998, 14:35-38.

6. Pozio E, La Rosa G, Gomez Morales MA: Epidemiology of human and animal trichinellosis in Italy since its discovery in 1887. Parasite 2001, 8:106-S108.

7. The International Trichinella Reference Center: [www.iss.it/site/Trichinella/index.asp]

8. European Food Safety Authority: Community Summary Report on trends and sources of zoonoses, zoonotic agents, antimicrobial resistance and food-borne outbreaks in the European Union in 2005. EFSA J 2006, 94:2-288.

9. European Food Safety Authority: Community Summary Report on trends and sources of zoonoses, zoonotic agents, antimicrobial resistance and food-borne outbreaks in the European Union in 2006. EFSA J 2007, 130:2-352.

10. European Food Safety Authority: Community Summary Report on trends and sources of zoonoses and zoonotic agents in the European Union in 2007. EFSA J 2009, 223:3-320

11. European Food Safety Authority: Community Summary Report on trends and sources of zoonoses and zoonotic agents and food-borne outbreaks in the European Union in 2008. EFSA J 2010, 8:1496.

12. European Food Safety Authority: Community Summary Report on trends and sources of zoonoses and zoonotic agents and food-borne outbreaks in the European Union in 2009. EFSA J 2011, 9:2090.

13. European Food Safety Authority: Community Summary Report on trends and sources of zoonoses and zoonotic agents and food-borne outbreaks in the European Union in 2010. EFSA J 2012, 10:2597.

14. European Food Safety A: The European Union Summary Report on Trends and Sources of Zoonoses, Zoonotic Agents and Food-borne Outbreaks in 2011. EFSA J 2013, 11:3129.

15. Gamble HR, Pozio E, Bruschi F, Nöckler K, Kapel CMO, Gajadhar AA: International Commission on Trichinellosis: recommendations on the Use of Serological Tests for the Detection of Trichinella Infection in Animals and Man. Parasite 2004, 11:3-13. 
16. Nöckler K, Serrano FJ, Boireau P, Kapel CM, Pozio E: Experimental studies in pigs on Trichinella detection in different diagnostic matrices. Vet Parasitol 2005, 132:85-90.

17. Gómez-Morales MA, Ludovisi A, Pezzotti P, Amati M, Cherchi S, Lalle M, Pecoraro F, Pozio E, Ring Trial Participants: International ring trial to detect anti-Trichinella IgG by ELISA on pig sera. Vet Parasitol 2009, 166:241-248.

18. Gómez-Morales MA, Ludovisi A, Amati M, Blaga R, Zivojinovic M, Ribicich M, Pozio E: A distinctive Western blot pattern to recognize Trichinella infections in humans and pigs. Int J Parasitol 2012, 42:1017-1023.

19. Nockler K, Reckinger S, Broglia A, Mayer-Scholl A, Bahn P: Evaluation of a western blot and ELISA for the detection of anti-Trichinella-IgG in pig sera. Vet Parasitol 2009, 163:341-347.

20. Marinculic A, Rapic D, Brglez J, Dzakula N, Stojiljkovic D: Epidemiological survey of trichinellosis in Yugoslavia. Southeast Asian J Trop Med Public Health 1991, 22S:302-307.

21. Nöckler K, Voigt WP, Protz D, Miko A, Ziedler K: Intravitale Diagnostik der Trichinellose beim Schwein mit dem indirekten ELISA. Berl Munch Tierarztl Wochenschr 1995, 108:167-174.

22. Robert F, Weil B, Kassis N, Dupouy-Camet J: Investigation of immunofluorescence cross-reactions against Trichinella spiralis by Western blot (immunoblot) analysis. Clin Diagn Lab Immunol 1996, 3:575-577.

23. Frey CF, Schuppers ME, Nöckler K, Marinculić A, Pozio E, Kihm U, Gottstein B: Validation of a Western Blot for the detection of anti-Trichinella spp. antibodies in domestic pigs. Parasitol Res 2009, 104:1269-1277.

24. Pozio E, Cossu P, Marucci G, Amati M, Ludovisi A, Gomez Morales MA, La Rosa G, Firinu T: The birth of a Trichinella britovi focus on the Mediterranean island of Sardinia (Italy). Vet Parasitol 2009, 159:361-363.

25. Pozio E, La Rosa G: Trichinella. In Molecular Detection of Foodborne Pathogens. Edited by Liu D. Boca Raton: CRC Press; 2010:851-863 [Taylor \& Francis Group].

26. World Organisation for Animal Health: Principles of validation of diagnostic assays for infectious diseases. In Manual of Diagnostic Tests and Vaccines for Terrestrial Animals. Edited by World Organization for Animal Health. Paris: World Health Organization; 2008:34-45.

27. Gamble HR, Anderson WR, Graham CE, Murrell KD: Diagnosis of swine trichinosis by enzyme-linked immunosorbent assay (ELISA) using an excretory-secretory antigen. Vet Parasitol 1983, 13:349-361.

28. Nöckler K, Kapel CMO: Detection and surveillance for Trichinella: meat inspection and hygiene, and legislation. In FAONHO/OIE Guidelines for the Surveillance, Management, Prevention and Control of Trichinellosis. Edited by Dupouy-Camet J, Murrell KD. Paris: World Health Organization; 2007:69-97 [World Organization for Animal Health].

29. Murrell DK: Trichinella spiralis: acquired immunity in swine. Exp Parasitol 1985, 59:347-354.

30. Marinculic A, Gamble HR, Urban JF, Rapic D, Zivicnjak T, Smith HJ, Murrell $\mathrm{KD}$ : Immunity in swine inoculated with larvae or extracts of a pig isolate and a sylvatic isolate of Trichinella spiralis. Am J Vet Res 1991, 52:754-758.

31. Pozio E: Searching for Trichinella: not all pigs are created equal. Trends Parasitol 2014, 30:4-11

32. Cuttell L, Gómez-Morales MA, Cookson B, Adams PJ, Reid SA, Vanderlinde PB, Jackson LA, Gray C, Traub RJ: Evaluation of ELISA coupled with Western blot as a surveillance tool for Trichinella infection in wildboar (Sus scrofa). Vet Parasitol 2014, 199:179-190.

33. Boadella M, Barasona JA, Pozio E, Montoro V, Vicente J, Gortazar C, Acevedo P: Spatio-temporal trends and risk factors for Trichinella species infection in wild boar (Sus scrofa) populations of central Spain: a long-term study. Int J Parasitol 2012, 42:739-745.

34. Sandfoss M, DePerno C, Patton S, Flowers J, Kennedy-Stoskopf S: Prevalence of antibody to Toxoplasma gondii and Trichinella spp. in feral pigs (Sus scrofa) of eastern North Carolina. J Wildl Dis 2011, 47:338-343.

35. Merialdi G, Bardasi L, Fontana MC, Spaggiari B, Maioli G, Conedera G, Vio D, Londero M, Marucci G, Ludovisi A, Pozio E, Capelli G: First reports of Trichinella pseudospiralis in wild boars (Sus scrofa) of Italy. Vet Parasitol 2011, 178:370-373.

\section{doi:10.1186/1756-3305-7-17}

Cite this article as: Gómez-Morales et al:: Indirect versus direct detection methods of Trichinella spp. infection in wild boar (Sus scrofa). Parasites \& Vectors 2014 7:171.

\section{Submit your next manuscript to BioMed Central and take full advantage of:}

- Convenient online submission

- Thorough peer review

- No space constraints or color figure charges

- Immediate publication on acceptance

- Inclusion in PubMed, CAS, Scopus and Google Scholar

- Research which is freely available for redistribution

Submit your manuscript at www.biomedcentral.com/submit
C) Biomed Central 